\title{
PALEOBATIMETRIA DA MARGEM CONTINENTAL DO BRASIL DURANTE O ALBIANO
}

\author{
EDUARDO A.M. KOUTSOUKOS* e DIMAS DIAS-BRITO*
}

\begin{abstract}
Paleobathymetric estimations obtained from paleoecological researches carried out in various Brazilian marginal basins reveal that during the Albian all this portion of the South Proto-Atlantic was practically on the same paleogeographic setting, where the sediment accumulation took place in a neritic environment. During the Early-Middle Albian, extensive and thick carbonate deposits were formed in very shallow to middle neritic environments $(0-50 \mathrm{~m}$ of water-depth), corresponding to the first pre-oceanic marine deposition over the spreading South Atlantic. The Late Albian shows a change in the oceanographic and depositional conditions, with an increase of the sea-level to a deep neritic environment $(100-200 \mathrm{~m})$. The comparison of the paleoecological results from the Brazilian margin with those coming from other areas of the South Atlantic suggests that during the Albian this ocean was a narrow and shallow epicontinental sea.
\end{abstract}

INTRODUÇÃo Os estudos paleoecológicos realizados no Centro de Pesquisas da Petrobrás, com base em foraminíferos e a partir de centenas de poços perfurados ao longo da extensa margem atlântica brasileira, revelam que as condições paleoceanográficas durante o Albiano foram relativamente uniformes e generalizadas. Considerando-se, por outro lado, os dados e as interpretações disponíveis na literatura, chega-se à conclusão de que esta uniformidade não se restringiu somente à margem brasileira, mas a todo o Atlântico Sul albiano. Este trabalho objetiva a apresentação deste conjunto de evidências, mostrando as peculiaridades paleobatimétricas e oceanográficas desta fase primeva do Atlântico Sul.

ESTUDOS ANTERIORES Entre os trabalhos de paleoecologia que fornecem dados paleobatimétricos concernentes à fase albiana da margem atlântica brasileira, citam-se: Beurlen (1982), Dias-Brito (1982, 1985a, 1985b, 1987), Koutsoukos (1982, 1984, 1987), Viviers (1982, 1985, 1987), Viviers \& Regali (1987), Azevedo (1983), Estrella et al. (1984) e Azevedo et al. (1987).

\section{- ALBIANO NA MARGEM ATLÃNTICA BRASILEI-}

RA Os depósitos albianos na margem continental do Brasil constituem o registro da fase inicial do Atlântico Sul nesta área. Assentam-se, em grande parte, sobre camadas saliferas (margem leste) e constituem, neste segmento, espessos pacotes carbonáticos. Na margem equatorial, as acumulações albianas variaram sua natureza litológica, oscilando entre carbonatos e terrígenos (Fig. 1).

A análise detalhada de sua paleofauna e paleoflora revela que situações fisiográficas especiais, combinadas com baixos valores batimétricos e relativa estabilidade tectônica, devem ter ocorrido neste tempo e, como resultado, dando origem a um longo e estreito mar epicontinental. Um esquema geral dos ambientes ao longo do Albiano, construído com base

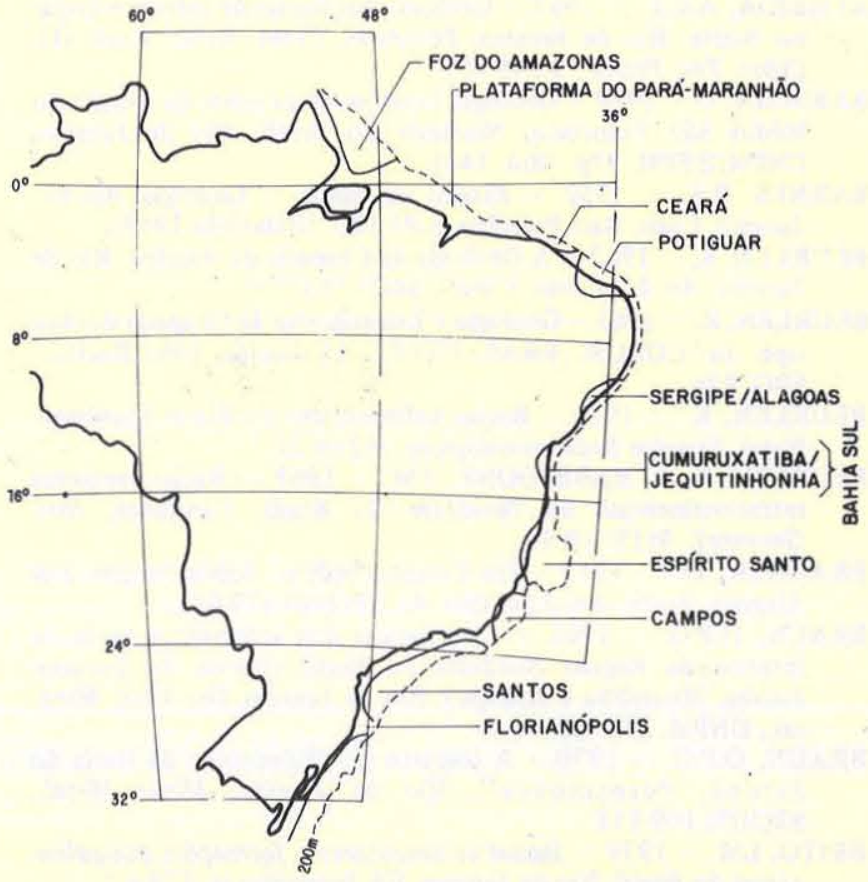

Figura 1 - Mapa de situação das principais bacias marginais brasileiras consideradas neste trabalho (adaptação de Ojeda 1982)

nos dados paleobatimétricos fornecidos por estudos de foraminíferos, é apresentado na figura 2. A relação e a caracterização das principais assembléias de foraminíferos e de alguns outros grupos orgânicos, bem como sua distribuição paleoambiental (relação microfósseis/habitat), são resumidas nos quadros de 1 a 7 .

De uma maneira geral, o detalhamento biocronoestratigráfico permite a individualização de duas fases distintas na seqüência albiana: eo-mesoalbiana e neoalbiana. 


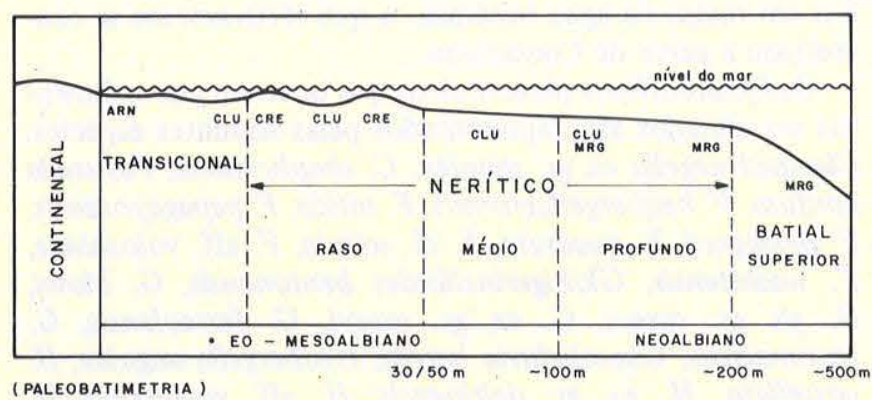

Figura 2 - Modelo esquemático hipotético dos ambientes marinhos do: Albiano da margem continental brasileira $(C R E=$ calcarenito; $C L U=$ calcilutito $; M R G=$ marga $;$ $A R N=$ arenito)

Quadro 1 - Assembléias microfossiliferas das Bacias de Florianópolis e Santos

\begin{tabular}{|l|l|}
\hline $\begin{array}{c}\text { PALEOAMBENE } \\
\text { OEPOSICIONAL }\end{array}$ & ASSEMBLÉIAS DE MICROFÓS SE IS \\
\hline
\end{tabular}

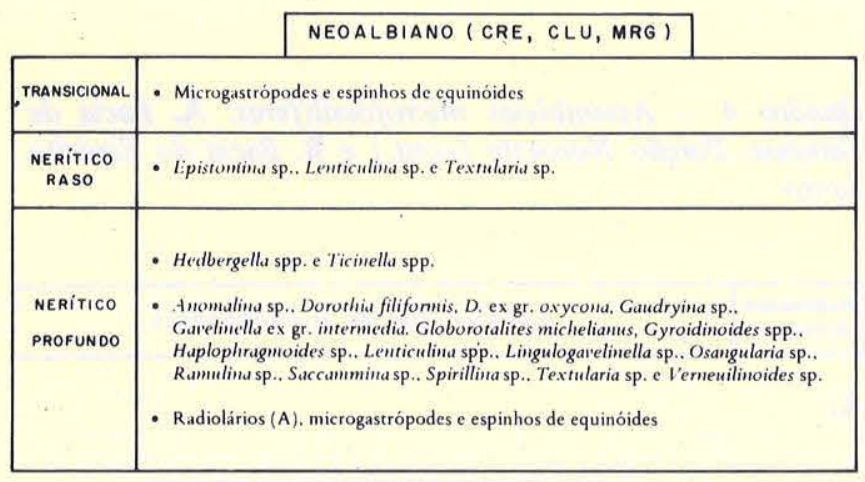

\begin{tabular}{|c|c|}
\hline & EO-MESOALBIANO (CRE, CLU) \\
\hline $\begin{array}{l}\text { TRANSICIONAL } \\
\text { CONTINENTAL }\end{array}$ & - Oogônias de algas carófitas e ostracodes. \\
\hline $\begin{array}{l}\text { NERÍTICO } \\
\text { RASO }\end{array}$ & $\begin{array}{l}\text { - Far'usella spp. } \\
\text { - Epistomina sp. } \\
\text { - Microgastrópodes, micropelecipodes, ostracodes e espinhos de equinóides }\end{array}$ \\
\hline $\begin{array}{l}\text { NERITICO } \\
\text { RASO } \\
\text { MÉOIO }\end{array}$ & $\begin{array}{l}\text { - Farusella spp. (F) e Hedbergella spp. (F/A) } \\
\text { - Dorothia ex gr. oxycona. Gavelinella ex gr. internedia e Lenticulina spp. } \\
\text { - Ostracodes (Conchoecia sp.), radiolários (R). microgastrópodes e espinhos } \\
\text { de equinóides. }\end{array}$ \\
\hline
\end{tabular}

\begin{tabular}{|c|c|c|}
\hline \multicolumn{3}{|c|}{ LEGENDA } \\
\hline$R=R A R$ & $A=A B U N D A N T E$ & $M R G=M A R G A$ \\
\hline$c=\operatorname{com}$ & CRE = CALCARENITO & ARN $=$ ARENITO \\
\hline$F=F$ REQÜENTE & CLU $=$ CALCILUTITO & $F L H=F O L H E L H O$ \\
\hline
\end{tabular}

A fase eo-mesoalbiana Representando os primeiros momentos da fase pré-oceânica, que se prolongou pelo tempo Cenomaniano-Turoniano, esses sedimentos são constituídos, predominantemente, por calcarenitos/calcilutitos. Nas áreas proximais, têm-se fácies mistas de carbonatos e terrígenos. Em todas as bacias estudadas, o conteúdo microfossilífero claramente aponta para condições de mar tropical, restrito e raso (valores paleobatimé- tricos entre 0 e $50 \mathrm{~m}$ ), com anomalia no piso marinho. Esta anomalia, evidenciada pela monotonia, baixa diversidade e baixa freqüência faunística de fundo, tem sido interpretada como resultante de hipersalinidade nas águas do estreito mar epicontinental (Dias-Brito 1982). Os quadros mencionados exibem as assembléias microfossilíferas que caracterizam a paleobatimetria desta fase. Entre os foraminiferos planctônicos, citam-se: Favusella spp., Globigerinelloides barri, G. ex gr. caseyi, G. gyroidinaeformis, Hedbergella ex gr. delrioensis, $H$. aff. gorbachikae, $H$. luterbacheri, $H$. aff. maslakovae, $H$. aff. trocaoidea, Heterohelix moremani, Ticinella bejaouensis, $T$. aff. primula, T. roberti, Whiteinella spp., entre os calcisferulídios são constatadas as espécies Pithonella sphaerica (freqüênte) e Pithonella ovalis (ocorrência esporádica).

A fase neoalbiana Os sedimentos neoalbianos, em geral, têm caráter pelítico (calcilutitos e margas), revelando que as condições paleobatimétricas se alteraram em relação à fase anterior. $\mathrm{O}$ aumento na espessura na lâmina de água do mar albiano é registrado praticamente em todas as bacias, o que é constatado consistentemente pela análise da paleobiota. Verifica-se um incremento na freqüência e

Quadro 2 - Assembléias microfossiliferas da Bacia de Campos; Porção Centro-oriental

\begin{tabular}{|l|l|}
\hline PALEOAMBIEMTE & ASSEM BLÉIAS DE MICROFÓSȘEIS \\
\hline
\end{tabular}

\begin{tabular}{|c|c|}
\hline & VRACONIANO (CLU, MRG) \\
\hline $\begin{array}{l}\text { NERITICO } \\
\text { PROFUNDO }\end{array}$ & $\begin{array}{l}\text { - Clavihedbergella sp., Globigerinelloides spp., Hedbergella spp., } \\
\text { Praeglobotruncana sp., Ticinella spp., e Whiteinella spp. } \\
\text { - Anomalinoides sp., Astacolus sp., Conorboides? sp., Dentalina sp., Doroihia } \\
\text { levis, D. ex gr. oxycona, Frondicularia sp., Gavelinella ex gr. intermedia, } \\
\text { Globorotalites michelianus, Gyroidinoides spp., Lagena apiculata, } \\
\text { Lingulogavelinella sp., Lenticulina spp., Nodosaria sp., Osangularia utaturensis, } \\
\text { Pleurostomella obtusa, Ramulina sp., Reussella? sp., Saracenaria spp. e } \\
\text { Textularia sp. } \\
\text { - Radiolários }\end{array}$ \\
\hline $\begin{array}{l}\text { NERITICO } \\
\text { PROFUNDO } \\
/ \\
\text { BATIAL } \\
\text { SUPERIOR }\end{array}$ & $\begin{array}{l}\text { - Hedbergella spp., Praeglobotruncana sp. e Rotalipora sp. (F) } \\
\text { - Associaçào bentônica similar à anterior }\end{array}$ \\
\hline
\end{tabular}

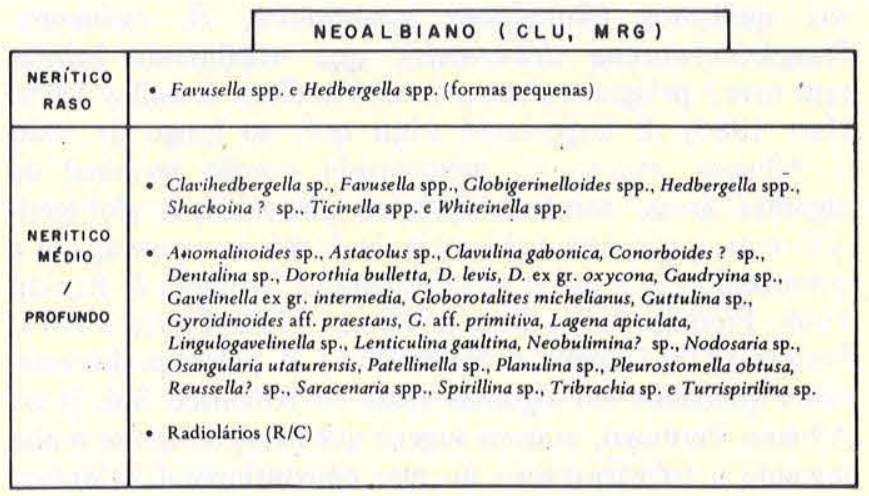


Quadro 3 - Assembléias microfossiliferas da Bacia de Campos; A. Porção Centro-Oriental (cont.) e B. Porção Noroeste

\begin{tabular}{|l|l|}
\hline $\begin{array}{r}\text { Paleoamiente } \\
\text { Deposicional }\end{array}$ & ASSEM BLÉIAS DE MICROFÓSSEIS \\
\hline
\end{tabular}

A.

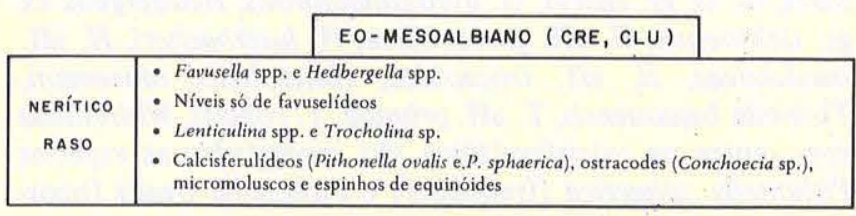

B.

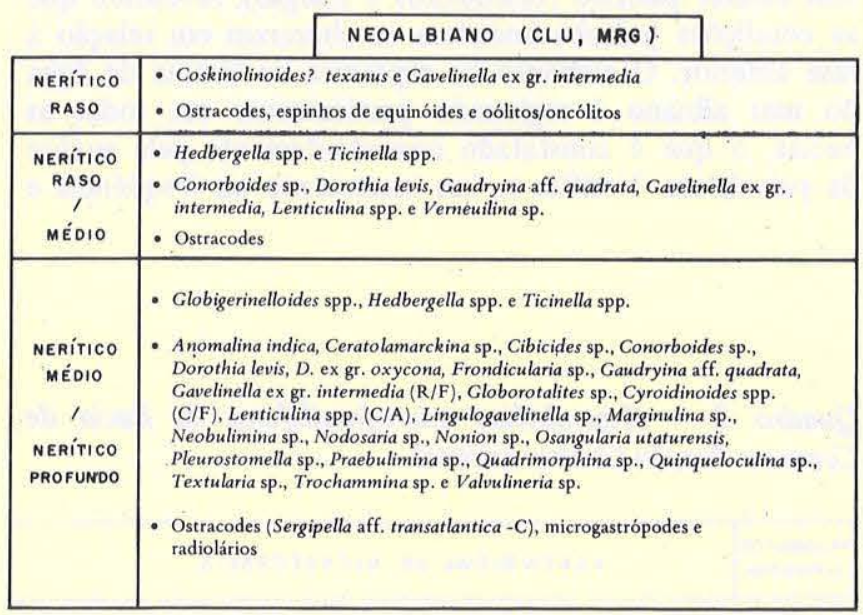

diversidade de foraminíferos bentônicos e planctônicos, de calcisferulídeos e na presença de nanofósseis calcários. Tal alteração biótica em relação à fase anterior é interpretada por Dias-Brito (1983a, b) como sendo derivada de uma elevação do nível marinho e do aumento no grau de contato entre os oceanos Atlântico Sul e Atlântico Norte, já iniciado na fase eo-mesoalbiana. Os valores paleobatimétricos de então estiveram em torno de 100 a $200 \mathrm{~m}$ e os maiores índices foram alcançados no Neoalbiano Terminal (Vraconiano). Em certas bacias, nesta faixa de tempo, é possível que determinadas áreas tivessem uma paleobatimetria um pouco mais profunda, já que são encontradas algumas espécies de foraminíferos quilhados (Rotalipora appenninica, $R$. ticinensis, Praeglobotruncana delrioensis), que usualmente habitaram níveis pelágicos abaixo de 200 m (Hart \& Bailey 1979, Hart 1980). É importante frisar que, ao longo de todo o Albiano, exceto na mencionada porção terminal de algumas áreas, somente ocorrem planctônicos globigeriniformes, que foram habitantes de domínios epipelágicos e colonizadores de mares epicontinentais (Douglas \& Rankin 1969, Frerichs 1971, Sliter 1972 a, 1972 b, Frerichs 1975, Petters 1978, Douglas \& Savin 1978). A presença dos referidos quilhados em algumas áreas do Atlântico Sul, já no Albiano Terminal, poderia sugerir que neste tempo se tenha iniciado a transformação do mar epicontinental Atlântico
Sul em massa de água oceânica, o que efetivamente se concretizou a partir do Coniaciano.

Os foraminíferos planctônicos que ocorrem nos sedimentos neoalbianos são representados pelas seguintes espécies: Clavihedbergella ex gr. simplex, C. simplicissima, Favusella confusa, $F$. hedbergellaeformis, $F$. nitida, $F$. papagayosensis, $F$. pessagnoi, $F$. quadrata, $F$. cf. scitula, $F$. aff. voloshinae, F. washitensis, Globigerinelloides bentonensis, G. blowi, $G$. ex gr. caseyi, G. ex gr. caseyi, G. ferreolensis, $G$. texomaensis, Guembelitria harrisi, Hedbergella angolae, $H$. costellata, $H$. ex gr. delrioensis, $H$. aff. gargasiana, $H$. gorbachikae, $H$. planispira, $H$. cf. roblesae, $H$. trocoidea, Heterohelix moremani, Praeglobotruncana delrioensis, Shackoina sp., Ticinella breggensis, $T$. cf. caronae, $T$. aff. digitalis, $T$. floresae, $T$. madecassiana, $T$. petalloidea, $T$. praeticinensis, $T$. primula, $T$. ex gr. raynaudi, $T$. roberti ¿ Whiteinella bornholmensis. As freqüentes formas bentônicas são mostradas nos quadros de 1 a 7 .

A evolução paleobatimétrica da margem atlântica brasileira durante o Albiano é esquematizada na figura 3. A quebra batimétrica sugerida para o limite eo-mesoalbiano-neoalbiano pode indicar um rápido incremento na lâmina de água do domínio nerítico ou ser indício de um hiato não-mensurável em termos de resolução biocronoestratigráfica.

Quadro 4 - Assembléias microfossiliferas: A. Bacia de Campos, Porção Noroeste (cont.) e B. Bacia do Espirito Santo

\begin{tabular}{|l|l|}
\hline PALEOAMBIENTE \\
DEPOSICIONAL
\end{tabular}$\quad$ ASSEM BLÉIAS DE MICROFÓSSEIS

A.

\begin{tabular}{|c|c|}
\hline & EO-MESOALBIANO (ARN, CRE, CLU) \\
\hline TRANSICIONAL & $\begin{array}{l}\text { - Haplophragmium? sp. e Haplophragmoides? sp. } \\
\text { - Oogônias de algas carófitas e megásporos carbonizados }\end{array}$ \\
\hline $\begin{array}{l}\text { NERÍTICO } \\
\text { RASO }\end{array}$ & $\begin{array}{l}\text { - Favusella spp. e Hedbergella spp. } \\
\text { - Anomalina indica, Dorothia levis Gavelinella ex gr. intermedia, Lenticulina spp. } \\
\text { e Trocholina sp. } \\
\text { - Acicularia (Dasycladaceae) sp. e oólitos/oncólitos }\end{array}$ \\
\hline $\begin{array}{l}\text { NERÍTICO } \\
\text { RASO } \\
\text { NERÍTICO } \\
\text { MÉDIO }\end{array}$ & $\begin{array}{l}\text { - Favusella spp. e Hedbergella spp. } \\
\text { - Ammobaculites sp., Anomalina indica (C/F), Dorothia levis, D. ex gr. oxycona, } \\
\text { Gavelinella ex gr. intermedia (C), Gyroidinoides spp., Lenticulina spp., Patellina } \\
\text { sp., Quinqueloculina? sp., Textularia sp. e Trocholina sp. } \\
\text { - Microgastrópodes e radiolários }\end{array}$ \\
\hline
\end{tabular}

B.

\begin{tabular}{|c|c|c|}
\hline & NEOALBIANO (CRE, CLU, MRG) & \\
\hline $\begin{array}{c}\text { NERÍTICO } \\
\text { RASO }\end{array}$ & \multicolumn{2}{|c|}{$\begin{array}{l}\text { - Favusella spp. e Guembelitria sp. } \\
\text { - Cibicides sp., Gavelinella ex gr. intermedia, Gyroidinoides spp. e Valvulineria sp. } \\
\text { - Oólitos/oncólitos (A) }\end{array}$} \\
\hline $\begin{array}{l}\text { NERITICO } \\
\text { MEDIO } \\
\text { NERÍTICO } \\
\text { PROFUNDO }\end{array}$ & \multicolumn{2}{|c|}{$\begin{array}{l}\text { - Clavihedbergella sp., Favusella spp., Globigerinelloides spp., Hedbergella spp., } \\
\text { Praeglobotruncana sp. e Ticinella spp. } \\
\text { - Gavelinella ex gr. intermedia, Globorotalites sp., Lenticulina spp. e } \\
\text { Osangularia sp. }\end{array}$} \\
\hline
\end{tabular}




\begin{tabular}{|c|c|c|c|c|}
\hline \multirow{2}{*}{ TEMPO } & \multirow{2}{*}{ ROCHA } & \multicolumn{2}{|c|}{ BATIMETRIA (m) } & \multirow{2}{*}{$\begin{array}{l}\text { CARACTERÍSTICAS } \\
\text { PALEOBIOTICAS }\end{array}$} \\
\hline & & 100 & 200 & \\
\hline $\begin{array}{l}\text { NEO- } \\
\text { ALBIANO }\end{array}$ & $\begin{array}{l}M R G \\
C L U\end{array}$ & i & & $\begin{array}{l}\text { - PRESENÇA SIGNIFICATIVA DE RADIOLÁRIOS } \\
\text { - ALTA FREQÜÊNCIA DE CALCISFERULÍDEOS } \\
\text { - ALTA FREQÜÊNCIA DE FORAMS. PLACTS. E } \\
\text { BENTS. } \\
\text { - MÉDIA / ALTA DIVERSIDADE ESPECÍFICA }\end{array}$ \\
\hline $\begin{array}{l}\text { EO- } \\
\text { MESO- } \\
\text { ALBIANO }\end{array}$ & $\begin{array}{l}C L U \\
C R E\end{array}$ & $\begin{array}{l}1 \\
1 \\
1 \\
1 \\
1 \\
1 \\
1 \\
1 \\
1 \\
1 \\
1\end{array}$ & & $\begin{array}{l}\text { - ESPORÁDICOS NÍVEIS RICOS EM PLANCTÔ- } \\
\text { NICOS MONOESPECÍFICOS } \\
\text { - RAROS CALCISFERULÍDEOS, RADIOLÁRIOS } \\
\text { - RAROS OSTRACODES, EQUINÓIDES, MICRO- } \\
\text { MOLUSCOS } \\
\text { - RAROS FORAMS. BENTÔNICOS E PLANCTÔNI } \\
\text { COS } \\
\text { - BAIXA DIVERSIDADE ESPECÍFICA }\end{array}$ \\
\hline
\end{tabular}

Figura 3 - Evolução paleobatimétrica generalizada da margem atlântica brasileira durante o Albiano

Quadro 5 - Assembléias microfossiliferas: A. Bacia de Cumuruxatiba/Jequitinhonha (Bahia Sul); B. Bacia de Sergipe/Alagoas

\begin{tabular}{|l|l|}
\hline $\begin{array}{c}\text { PALEOMGaEmTE } \\
\text { DE POSICIOMAL }\end{array}$ & ASSEM BLÉIAS DE MICROFÓSSEIS \\
\hline
\end{tabular}

A.

ALBIANO (CRE, CLU)

\begin{tabular}{|c|l}
\hline NE RÍtIco & - Favusella spp. e Hedbergella spp. \\
RASO & - Óólitos/oncólitos (A) \\
\hline
\end{tabular}

B.

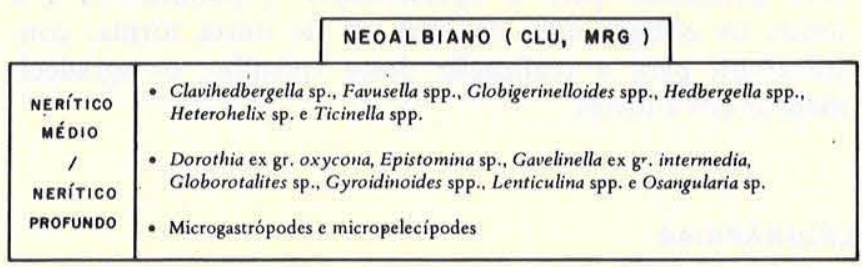

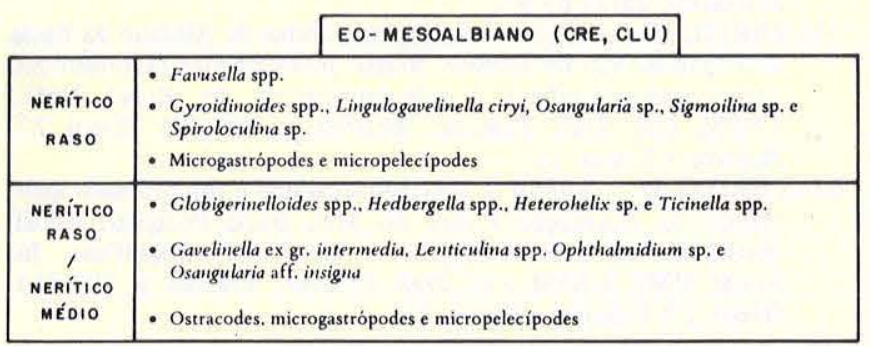

Quadro 6 - Assembléias microfossiliferas: A. Bacia Potiguar; B. Bacia do Ceará

\begin{tabular}{|c|c|}
\hline $\begin{array}{l}\text { PLL EOAMAIEUTE } \\
\text { DEP OSICIOMAL }\end{array}$ & A SSEMBLÉIAS DE MICROFÓSSEIS \\
\hline
\end{tabular}

A.

\begin{tabular}{|c|c|c|}
\hline & & EO-MESOALBIANO (CLU, MRG) \\
\hline $\begin{array}{l}\text { NERITICO } \\
\text { RASO }\end{array}$ & $\begin{array}{l}\text { - Favusella spp. } \\
\text { - Nodosarídeos e text } \\
\text { - Ostracodes (Aracaju } \\
\text { microgastrópodes }\end{array}$ & $\begin{array}{l}\text { tularídeos } \\
\text { uia benderi), calcisferulídeos (Pithonella sphaerica) e }\end{array}$ \\
\hline
\end{tabular}

B.

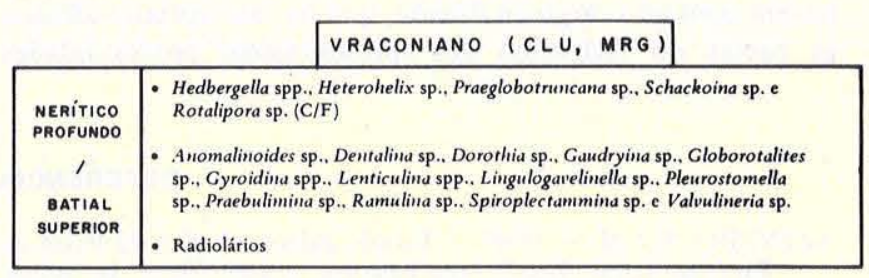

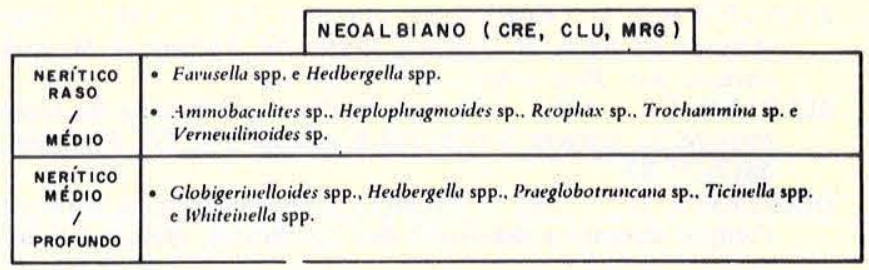


Quadro 7 - Assembléias microfossiliferas: A. Bacia do Ceará (cont.); B. Bacia da Foz do Amazonas; C. Plataforma do Pará-Maranhão

\begin{tabular}{|l|l|}
\hline $\begin{array}{c}\text { PALEOAMBiEMTE } \\
\text { OEPOSICIOHAL }\end{array}$ & ASSEM BLÉIAS DE MICROFÓSSEIS \\
\hline
\end{tabular}

A.

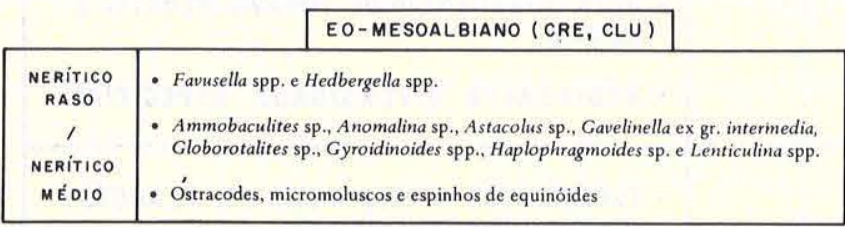

B.

\begin{tabular}{|c|c|c|}
\hline t & NEOALBIANO ( CRE, CLU) & \\
\hline $\begin{array}{l}\text { NERITICO } \\
\text { RASO } \\
\text { MÉDIO }\end{array}$ & $\begin{array}{l}\text { - Favusella spp., Globigerinelloides spp., Hedbergella spp. c } \\
\text { - Microgastrópodes e micropelecípodes }\end{array}$ & eterohelix sp. \\
\hline
\end{tabular}

C.

EO-MESOALBIANO (CRE, CLU,FLH)

\begin{tabular}{|c|l|}
\hline $\begin{array}{c}\text { NERITICO } \\
\text { RASO } \\
\text { MÉOIO }\end{array}$ & • Globigerinelloides spp., Hedbergella spp. e Heterohelix sp. \\
\hline
\end{tabular}

\section{O ALBIANO EM OUTRAS ÁREAS DO ATLÂNTICO}

SUL Os dados disponíveis a respeito das condições paleobatimétricas em outras áreas do Atlântico Sul derivam, principalmente, das pesquisas do Deep Sea Drilling Project (DSDP).

Em 1973, Sclater \& McKenzie propuseram uma primeira reconstrução paleobatimétrica para o Atlântico Sul. Esses dois autores, com base em estudos envolvendo resfriamento de crosta e formação de bacias, propuseram que, entre a quebra inicial dos continentes sul-americano e africano e $75 \mathrm{Ma}$, as quatro grandes bacias então formadas tinham profundidades abaixo de $4.000 \mathrm{~m}$. Van Andel et al. (1977), ao estimarem a profundidade da zona de compensação da calcita (CCD) para o Aptiano-Albiano da área meridional do Atlântico Sul, sugerem uma paleobatimetria em torno de $3.000 \mathrm{~m}$. Por outro lado, os estudos geotectônicos feitos por Melguen et al. (1978) levaram os autores a considerar, porém com alta carga de dúvida, que na fase aptiano-albiana as bacias do Atlântico Sul apresentassem profundidades acima de 3.000 m. Ainda em 1978, Melguen apresentou uma curva paleobatimétrica para os sites 363 e 364 , onde os depósitos albianos são apresentados como tendo sido depositados em águas neríticas.

Em termos paleobiológicos e paleoecológicos, os dados sugerem águas rasas para a fase albiana. Wiedmann \& Neugebauer (1978), ao estudarem a forma de amonóides do Site 364 , assinalam que esses fósseis estão relacionados a ambiente nerítico interno ou epicontinental. Scheibnerová (1981), ao fazer uma síntese comparativa dos foraminíferos bentônicos albo-cenomanianos encontrados nas Legs 26, 36 e 40, considera que, neste tempo, o Atlântico Sul se apresentava com profundidades médias em torno de $100 \mathrm{~m}$, não excedendo, em locais mais profundos, a 300-400 m.

CONCLUSÕES As análises paleoecológicas feitas no Centro de Pesquisas da Petrobrás, com base em foraminíferos e a partir de centenas de poços perfurados na margem atlântica brasileira, evidenciam que a sedimentação albiana neste segmento do Atlântico Sul ocorreu sob condições paleobatimétricas relativamente uniformes. Os valores paleobatimétricos estiveram dominantemente vinculados a ecossistemas neríticos, tendo-se diferenciado duas fases:

a. Eo-mesoalbiana - As condições do meio não foram mais profundas que $50 \mathrm{~m}$.

b. Neoalbiana - A paleobatimetria da margem continental do Brasil esteve em torno de 100 a $200 \mathrm{~m}$. No momento geo-histórico do Neoalbiano Terminal (Vraconiano), podem ter ocorrido algumas áreas um pouco mais profundas com 200 a $300 \mathrm{~m}$.

A mudança paleobatimétrica verificada entre as duas fases é considerada resultante de um abrupto incremento da lâmina de água nerítica ou derivada da falta de registro sedimentar, não detectado a nível de resolução biocronoestratigráfica.

A comparação dos dados paleoecológicos obtidos para a faixa atlântica brasileira com aqueles obtidos em outras áreas do Atlântico Sul resulta na concepção, aqui apresentada, de que o referido oceano era, no Albiano, um mar epicontinental estreito e com relativa estabilidade tectônica Sensu strictu. A espessa sedimentação dominantemente carbonática deste tempo deve ter ocorrido pelo jogo sedimentação/subsidência, em cuja dinâmica a movimentação halocinética teve participação destacada.

Agradecimentos À Diretoria Executiva da Petrobrás, pela permissão para a apresentação e publicação; e a todos os colegas que, de uma ou de outra forma, contribuíram para a realização deste trabalho, os agradecimentos dos autores.

\section{REFERÊNCIAS BIBLIOGRÁFICAS}

AZEVEDO, R.L.M. - 1985 - Estudo paleoecológico da Bacia do Espírito Santo, Brasil, com base em foraminíferos. In: Brasil, MME, DNPM, Col. Trab. Paleont., Brasília, p. 261-271. (Geol. 27. Paleont. e Estrat. 2).

AZEVEDO, R.L.M.; GOMIDE, J.; VIVIERS, M.C. - 1987 - Geo-história da Bacia de Campos, Brasil: do Albiano ao Maastrichtiano. Rev. Bras. Geoc., 17(2): 139-146.

BEURLEN, G. - 1982 - Bioestratigrafia e geo-história da seção marinha da margem continental brasileira. Bol. Téc. Petrobrás, 25(2):77-83.

DIAS-BRITO, D. - 1982 - Evolução paleoecológica da Bacia de Campos durante a deposição dos calcilutitos, margas e folhe- lhos da Formação Macaé (Albiano e Cenomaniano?). Bol. Téc. Petrobrás, 25(2):84-97.

DIAS-BRITO, D. - 1985a - Calcisphaerulidae do Albiano da Bacia de Campos, Rio de Janeiro, Brasil: investigações taxonômicas, biocronoestratigráficas e paleoambientais. In: Brasil, MME, DNPM, Col. Trab. Paleont., Brasília, p. 295-305. (Geol. 27. Paleont. e Estrat. 2).

DIAS-BRITO, D. - 1985b - Calcisphaerulidae e microfósseis associados da Formação Ponta do Mel, Bacia Potiguar, Brasil: considerações paleoecológicas e biocronoestratigráficas. In: Brasil, MME, DNPM, Col. Trab. Paleont., Brasília, p. 307-314. (Geol. 27. Paleont. e Estrat. 2). 
DIAS-BRITO, D. - 1987 - A Bacia de Campos no Mesocretáceo: uma contribuição à paleoceanografia do Atlântico Sul primitivo. Rev. Bras. Geoc., 17(2):162-167.

DOUGLAS, R.G. \& RANKIN, C - 1969 - Cretaceous planktonic foraminifera from Bornholm and their zoogeographic significance. Lethaia, 2:185-217.

DOUGLAS, R.G. \& SAVIN, S.M. - 1978 - Oxigen isotopic evidence for the depth stratification of Tertiary and Cretaceous planktic foraminifera. Marine Micropaleontology, 3:175-196.

ESTRELLA, G.O.; ROCHA MELLO, M.; GAGLIANONE, P.C.; AZEVEDO, R.L.M.; TSUBONE, K.; ROSSETTI, E.; CONCHA, J.; BRÜNING, I.M.R.A. - 1984 - The Espírito Santo Basin (Brazil) source rock characterization and petroleum habitat. In: DEMAISON, G. \& MURRIS, R.J. (eds.). Petroleum Geochemistry and Basin Evaluation. Tulsa, AAPG. (Memoir 35).

FRERICHS, W.E. - 1971 - Planktonic foraminifera in sediments of the Andaman sea. J. Foram. Research, 1:1-14.

FRERICHS, W.E - 1975 - Planktonic foraminifera from the chalky members of the Niobrara formation Centennial valley, Wyoming. J. Foram. Research, 5(4):294-312.

HART, M.B. - 1980 - A water depth model for the evolution of the planktonic Foraminiferida. Nature, 286:252-254.

HART, M.B. \& BAILEY, H.M. - 1979 - The distribution of planktonic Foraminiferida in the mid-Cretaceous of N.W. Europe. Aspekte der Kreide Europas. IUGS., Ser. A, 6:527-542.

KOUTSOUKOS, E.A.M. - 1982 - Geo-história e paleoecologia das bacias marginais de Florianópolis e Santos. In: CONGR. BRAS. GEOL., 32, Salvador, 1982. Anais... Salvador, SBG. v. 5, p. 2369-2382.

KOUTSOUKOS, E.A.M. - 1984 - Evolução paleoecológica do Albiano ao Maastrichtiano na área noroeste da Bacia de Campos, Brasil, com base em foraminíferos. In: CONGR. BRAS. GEOL. 33, Rio de Janeiro, 1984. Anais... Rio de Janeiro, SBG. v. 2, p. 685-698.

KOUTSOUKOS, E.A.M. - 1987 - A área noroeste da Bacia de Campos, Brasil, do Mesocretáceo ao Neocretáceo: Evolução paleomabiental e paleogeográfica pelo estudo de foraminíferos. Rev. Bras. Geol., 17(2): 168-172.

MELGUEN, M. - 1978 - Facies evolution, carbonate dissolution cycles in sediments from the Eastern South Atlantic (DSDP Leg 40) since the Early Cretaceous. In: BOLLI, H.M. et al. Initial Reports of the Deep Sea Drilling Project. Washington, U.S. Government Printing Office, 40:981-1024.
MELGUEN, M.; LE PICHON, S.; SINGUET, J.C. - 1978 Paléoenvironnement de l'Atlantique Sud. Bull. Soc. Geol. Fr., 4:471-489.

PETTERS, S.W. - 1978 - Mid-Cretaceous paleoenvironments and biostratigraphy of the Benue Through, Nigeria. Geological Society of America Bulletin, 89:151-154.

SCHEIBNEROVÃ, V. - 1981 - Paleogeographical implicatons of Cretaceous benthic foraminifera recovered by the Deep Sea Drilling Project in the Western South Atlantic. Cretaceous Research, 2(1):1-18.

SCLATER, J.G. \& McKENZIE, D.P. - 1973 - Paleobathymetry of the South Atlantic. Geol. Soc. America Bull., 84(10): 3202-3216.

SLITER, W.V. - 1972a - Cretaceous foraminifers depth habitats and their origin. Nature, 239:514-515.

SLITER, W.V. - 1972b - Upper cretaceous planktonic foramiferal zoogeography and ecology-eastern pacific margin. Paleogeogr., Paleoclimatol., Paleoecol., 12:15-31.

VAN ANDEL, T.H.; THIEDE, J.; SCLATER, J.G.; HAY, W.W. 1977 - Depositional history and paleoceanography of the South Atlantic Ocean during the last 125 million years. $J$. Geol., 85:651-698.

VIVIERS, M.C. - 1982 - Biocronoestratigrafia da Bacia do Ceará. In: CONGR. BRAS. GEOL., 32, Salvador, 1982. Anais. Salvador, SBG. v. 5, p. 2433-2449.

VIVIERS, M.C - 1985 - Características bioestratigráficas dos sedimentos albo/cenomanianos da Bacia do Ceará. Relações com outras bacias brasileiras e africanas. In: Brasil, MME, DNPM, Col. Trab. Paleont. Brasília, p. 529-538. (Geol. 27. Paleont. e Estrat. 2).

VIVIERS, M.C. \& REGALI, M.S.P. - 1987 - Estudo paleoambiental preliminar no Cretáceo da Bacia Potiguar. Rev. Bras. Geoc., 17(2): 122-130.

VIVIERS, M.C. - 1987 - Foraminíferos planctônicos do Cretáceo Médio, Bacia de Santos, Brasil. Rev. Bras. Geoc., 17(2):154-161.

WIEDMANN, J. \& NEUGEBAUER, J. - 1978 - Lower Cretaceous Ammonites from the South Atlantic Leg 40 (DSDP), their stratigraphic value and sedimentological properties. In: Init. Repts. DSDP. Washington, U.S. Government Printing Office, 40(suppl.):709-734.

MANUSCRITO 389

Recebido em 01 de setembro de 1986 Revisão aceita em 09 de abril de 1987

A idéia de excelência é intrínseca, como objetivo de qualquer atividade realizada com seriedade e, ainda mais, quando se trata de atividade intelectual. 